\title{
A Model of Growth Trajectory Bifurcation in Animals Ontogeny
}

\author{
V. L. Stass ${ }^{1}$ \\ ${ }^{1}$ Laboratory of Animal Genetics, Institute of Animal Husbandry and Veterinary Sciences, Latvia \\ Correspondence: V. L. Stass, Laboratory of Animal Genetics, Institute of Animal Husbandry and Veterinary \\ Sciences, Latvia. E-mail: valbet.axon@apollo.lv
}

Received: November 1, 2019

Accepted: November 25, 2019

Online Published: December 1, 2019

doi:10.5539/ijb.v12n1p20

URL: https://doi.org/10.5539/ijb.v12n1p20

\begin{abstract}
The aim of this study was to formulate and analyse a model of ontogenetic growth cessation in pigs. The cessation of growth when an animal reaches its species-specific size in ontogeny is still a problem. Systemic factors that contribute to this process are unknown. The focus of the research is an analysis of the growth dynamic that explains some aspects of the problem.

The method applied to meet the purpose of the study was mathematical modelling. To enhance the understanding of the growth trajectory in ontogeny an analytical model of growth in pigs was built. The model was formulated as a hybrid dynamic system with discrete-time, and continuum equations. The novelty of the study is a concept of ontogenetic growth in the pig. Both a new modelling technique, and new variables are introduced. A central theme of the study is an analysis of the growth trajectory bifurcation, and a description of the two emerged growth trajectories. A reading of a normal form of bifurcation applied to the growth trajectory bifurcation has been offered.

The results suggest that ontogenetic growth in pigs is not continuous. The growth trajectory has bifurcation at the point the animals attain their individual maximum weight. At this point, two new growth trajectories emerge. On one trajectory, animals continue to grow till a species maximum weight is reached. On other trajectory, animals continue to live till obtainable life span is attained. The emerged trajectories are genetic channels that open the way to grow for the certain phenotypes. Ontogenetic growth stops when the feed conversion coefficient grows into infinity.
\end{abstract}

Keywords: Ontogenetic Growth, Growth Trajectory, Maximum Weight, Feed Conversion, Hybrid Model, Order Parameter, Pitchfork Bifurcation

\section{Introduction}

The aim of this study is to investigate some aspects of ontogenetic growth in pigs by analysing dynamic of growth from $30 \mathrm{~kg}$ live weight up to the maximum weight. To meet the study purpose, a hybrid mathematical model was built. The model concerns the nutritional aspects of growth. It was formulated by combining standard continuum methods, and discrete-time difference equations techniques. In the model, the following two focal variables are explicitly included in the formulation, an invariant of growth, and a feed conversion coefficient. In the study, functional relations between relevant variables found in the experiments and field observations have been analysed. It is shown that growth dynamic is neither smooth nor continuous; there is bifurcation of the initial growth trajectory that causes two new growth trajectories to emerge. The novelty of the study is an innovative concept of the growth dynamic, and an approach to analysing bifurcation of the growth trajectory in pigs' ontogeny.

\subsection{Growth in Animals}

In this study, ontogenetic growth in animals has been analysed. Age and size at maturity are arguably the two most important life history traits of animals. Variation in both traits has severe effects on fitness. Genetic, developmental, and physiological mechanisms that control age and size at maturity are for the most part unknown. Nutrition and hormones play obvious and well-established roles in growth, but the natural mechanisms that cause the cessation of growth when an animal reaches a species-specific size remain among the great puzzles in biology (Nijhout, 2015). Growth dynamic analyses of animals such as the pig, provides a biological model for an understanding of growth in mammals including humans. The pig is an excellent biomedical model for humans. The swine has been used as a major mammalian model for studies because of the similarity in size and physiology, in organ development and disease progression (Lunney, 2007). Understanding how growth in animal is controlled 
is a major goal of biological research for two reasons. First, from a medical perspective, it is important to understand what goes wrong in the numerous diseases that involve the loss of growth control. Second, from a wider biological viewpoint, it is important to understand how genes control animal size (Weinkove \& Leevers, 2000).

\subsection{Modelling Biological Processes}

Biological processes differ considerably from those that study physics. If physical science has well-established laws of inanimate nature then the life sciences have few, if any, recognized universal laws (Coveney et al., 2016). However, biological processes, while founded entirely upon physical laws, are not modelled in terms of them. Still, the both sciences develop by applying the same strategy: knowledge in the fields has been acquired by a means of experiments and models. Models or theories are needed to convert experimental data to knowledge (Brenner, 2010). It is well-recognised that in biology and medicine, data do not translate readily into understanding, let alone treatments (Coveney et al., 2016). Moreover, much of the logic of the interactions in living systems is implicit. It follows that beyond a certain degree of complexity, armchair (qualitative) thinking is not only inadequate for understanding such systems, it can even be misleading (Noble, 2002).

The typical reasons for constructing mathematical models are the following. The models enable predictions, act as guides for future experimentation, aid knowledge synthesis, and increase scientific understanding (Aumann, 2006). As pigs are well-known model animals in human physiology and medicine, the results of growth modelling in pigs can be useful to research on cancer, development, and longevity.

\subsection{Nonlinear Processes}

The physical world is mostly nonlinear. This observation is the warning to us not to make assumptions based on the traditional linear thinking (Stewart, 2011). In biological systems, interactions and processes are often nonlinear. In these cases, intuitive, verbal reasoning may be misleading; whereas mathematical techniques allow us to analyse the effects of many interacting biological processes (Schumacher et al., 2016). In biological systems, the nonlinearities enter through feedback mechanisms (Coveney \& Fowler, 2005). The growth in animals is reportedly driven by such mechanisms. Evidence suggests that progressive decline in growth results from a genetic programme that occurs in multiple organs and involves the down-regulation of a large set of growth-promoting genes. This genetic programme does not appear to be driven simply by time, but rather depends on growth itself, suggesting that the limit on adult body size is imposed by a negative feedback loop. Current findings suggest that in mammals, body growth is limited, at least in part, by a negative feedback loop (Lui \& Baron, 2011).

In biology and medicine, models have typically been constructed by applying differential equations technique. While the continuum models are quicker and easier to implement, it can be difficult to relate system parameters to measurable biophysical quantities (Osborne et al., 2010). A major difficulty with this approach is that all the parameters within these equations are not usually known. Assuming unknown parameters continue to exist, one empirical option is to 'tune' them to reproduce biologically reasonable behaviour, although such an ad hoc procedure is clearly unsatisfactory (Coveney \& Fowler, 2005). While modelling growth in animals, there is one more aspect to consider, namely a spatial heterogeneity of biological objects. Living organisms are heterogeneous systems, and this fact causes additional difficulty for modelling such objects. Moreover, growth has been identified as an important factor in the production of spatial heterogeneity since it can fundamentally change the observed dynamics of patterning mechanisms (Maini et al., 2012). It is essential to point out that differential equations fail to account for the emergence of large-scale inhomogeneities, and for the influence of inhomogeneity on the overall dynamic of biological systems; the problem is that the differential equation is not a convenient tool for this task (Louzoun et al., 2001).

\section{Materials and Methods}

The data set was obtained from experiments on growing domestic pigs, fed from $30 \pm 6 \mathrm{~kg}$ up to $96 \pm 4 \mathrm{~kg}$ live weight. The pigs were housed and fed under non-industrial conditions, either in a pig testing station or in research facilities. The animals were kept loose in groups of up to four to a pen, or individually in pens, fed a dry balanced feed with unlimited access to water contingent on the experiment design, ad libitum, or a constrained diet, in accordance with the current body weight, adjusting the feed quantity once a week. The experiments were performed in compliance with Declaration of Helsinki, National legislation, and institutional rules.

\subsection{The Model's Variables}

Let $M$ denote an individual animal current live weight, measured in kilograms.

$M=\left\{\mathrm{M} \in \mathbb{R}_{+} \mid 30 \leq M \leq 600\right\}$, an animal individual maximum weight, $M_{\mathrm{x}}=600 \mathrm{~kg}$. 
' $m$ ' is an animal initial considered weight, measured in kilograms, $m \leq M, m_{0}=30 \mathrm{~kg}$.

Let $t$ denote the chronological discrete current time, measured in days from animals' birth.

$t=\{t \in \mathbb{N} \mid 0 \leq t<\infty\}, \Delta t=1,2,3, \ldots, \mathrm{n} . \mathrm{n} \in \mathbb{N}$.

' $t_{0}$ ' is time related to $m_{\mathrm{o}}, t_{\mathrm{o}}=90$ days.

Let $K$ denote the invariant of growth, nondimensional.

$K=\left\{K \in \mathbb{R}_{+} \mid 1 \leq K<11\right\}, K_{0}=1$.

Let $Z$ denote the current feed conversion coefficient, nondimensional.

$\mathrm{Z}=\left\{\mathrm{Z} \in \mathbb{R}_{+} \mid \mathrm{Z}_{\mathrm{o}} \leq \mathrm{Z} \leq \infty\right\},(\mathrm{Z}=\infty) \rightarrow\left(\mathrm{M}=\mathrm{M}_{\mathrm{x}}\right) \mathrm{V}\left(\mathrm{M}=\mathrm{M}_{\mathrm{xx}}\right)$.

\subsection{Hybrid Model}

In this study, two aspects of animal growth modelling have been taken into consideration: nonlinearity of the growth process, and heterogeneity of growing organisms. To address these aspects in the best possible way, a hybrid dynamic model was built. In broad, informal terms, a dynamic system is any collection of mathematical rules that determines the future behaviour of some system in terms of its state at any instant. More specifically, we consider two basic types of dynamic system: discrete, and continuous. In other words, a discrete dynamical system is a difference equation, and a continuous dynamic system is a differential equation, both kinds are deterministic (Stewart, 2011). Hybrid models have been built for systems with a mixture of continuous, and discrete-time dynamic. The models have often been used to capture the behaviour of systems that contain some sort of discontinuous events (Di Bernardo \& Hogan, 2010). Mathematically, these systems entail continuous, and discrete-state or discrete-time variables. Mathematical modelling by hybrid dynamical systems is particularly important for understanding the nonlinear dynamic of biological and medical systems (Aihara \& Suzuki, 2010). This reasoning suggests a possible explanation for the efficiency of discrete-time modelling technique. If we are to have chance to build an analytical model of ontogenetic growth, we need to consider diverse options; one option is a hybrid, discrete-time model that can outline the coarse dynamics of the same process without taking into consideration some implicit events that take place in the chosen discrete time unit. When the coarse outlines of the growth are clarified, a more detailed analyses could be applied. An advantage of this modelling technique is that this kind of models have no requirements as to the structure of the object or process.

The model does not entail unknown, speculative parameters. In the model, only functional relations between variables that follow from the analyses of the data set have been considered. Some relevant aspects are addressed by applying the modelling technique.

\section{Results}

A detailed analysis of the data set has revealed the following mathematical relations between variables. The growth invariant ' $\mathrm{K}$ ' has the following form:

$$
K=\frac{M t}{m_{o}\left(2 t-t_{o}\right)}
$$

Parameter $\mathrm{K}$ is the same for the same weight animals regardless of their daily gain. In general form, the functional relations between variables are given by system (2).

$$
\left\{\begin{array}{l}
\frac{M}{m_{o}}=2 K-1+\frac{\left(t-K t_{o}\right)}{t} \\
\frac{\left(t-K t_{o}\right)}{t}=\frac{(Z-2 K)(K-1)}{Z K}
\end{array}\right.
$$

Equation (1) and system (2) are mathematical functions that follow from the analyses of the data set.

\subsection{Growth up to Individual Maximum Weight, $M_{x}$}

From equation (1) and system (2) the following two growth equations have been derived.

$$
\begin{gathered}
\frac{1}{\mathrm{~m}_{\mathrm{o}}} \cdot \frac{\Delta \mathrm{M}}{\Delta \mathrm{t}}=\frac{1}{\mathrm{t}} \cdot \frac{\mathrm{ZK}(2 \mathrm{~K}+1)-2 \mathrm{~K}^{2}}{\mathrm{Z}(\mathrm{K}+1)-2 \mathrm{~K}}, \mathrm{~m}_{\mathrm{o}} \leq \mathrm{M} \leq \mathrm{M}_{\mathrm{x}} \\
\frac{1}{\mathrm{~m}_{\mathrm{o}}} \cdot \frac{\Delta \mathrm{M}}{\Delta \mathrm{t}}=\frac{1}{\mathrm{t}} \cdot \frac{\mathrm{m}_{\mathrm{o}}[\mathrm{Z}(2 \mathrm{~K}+1)-2 \mathrm{~K}]}{\mathrm{m}_{\mathrm{o}}[\mathrm{Z}(2 \mathrm{~K}+1)-2 \mathrm{~K}]-\mathrm{MZ}}, \mathrm{m}_{\mathrm{o}} \leq \mathrm{M} \leq \mathrm{M}_{\mathrm{x}},
\end{gathered}
$$


where $\mathrm{M}_{\mathrm{x}}$ denote an animal's individual maximum weight. Under the model conditions, this weight is $600 \mathrm{~kg}$. To find out the corresponding $\mathrm{K}_{\mathrm{x}}$ let us consider the following limit.

$$
\lim _{M \rightarrow M_{x}}\left(\frac{2 K-1}{K+1}\right)=\sqrt{3} \text {, where }\left.\left(K \rightarrow K_{x}\right)\right|_{M \rightarrow M_{x}}
$$

From (5) it follows that numerically, $K_{x}=5+3 \sqrt{3}=10,19615 . K_{x}$ corresponds to $M_{x}$, and $t_{x}=6,408$ years (Stass, 2019). The next task is to find $Z_{x}$ that corresponds to $K_{x}$ and $M_{x}$. From system (2) one can derive the following equation

$$
\frac{1}{m_{o}} \cdot \frac{\Delta M}{\Delta K}=\frac{2 K+1}{K}-\frac{2}{Z}, m_{o} \leq M \leq M_{x}
$$

Substituting $M_{x}$ and $K_{x}$ into (6) we have $Z_{x}=62,51$.

When individual maximum weight, $\mathrm{M}_{\mathrm{x}}$ is attained, animals' growth stops. As a result, the feed conversion coefficient, $Z_{x}$ grows into infinity $\left(Z_{x} \rightarrow \infty\right)$. We can find growth rate under this condition. Let us consider equation (3). Taking limit $\left(Z_{x} \rightarrow \infty\right)$ in (3) we have

$$
\lim _{Z \rightarrow \infty}\left(\frac{\Delta M}{\Delta t}\right)=\frac{m_{o}}{t_{x}} \cdot \frac{K_{x}\left(2 K_{x}+1\right)}{K_{x}+1}, \text { where }\left.\left(K=K_{x}\right)\right|_{Z \rightarrow \infty}
$$

In a more convenient form, limit (7) is given by

$$
\frac{1}{m_{o}} \cdot \frac{\Delta M_{x}}{\Delta t_{x}}=\frac{1}{t_{x}} \cdot \frac{K_{x}\left(2 K_{x}+1\right)}{K_{x}+1}
$$

In equation (7.1) $\mathrm{M}=\mathrm{M}_{\mathrm{x}}, \mathrm{K}=\mathrm{K}_{\mathrm{x}}$, and $\mathrm{Z}=\infty$. Equation (7.1) describes growth rate in the animals, which have not only reached their maximum individual weight, $\mathrm{M}_{\mathrm{x}}$ but have also passed through the bifurcation point and took on the growth trajectory $\left.\left.M_{x}\right|_{k=k_{x}} \rightarrow \quad M_{x}\right|_{k=k_{1}}$, where $\mathrm{K}_{1}=10,04975 . \mathrm{K}_{1}$ corresponds to $\mathrm{t}_{1}$, the species obtainable life span, $\mathrm{t}_{1}=24,90$ years (Stass, 2019).

\subsection{Growth Trajectory Bifurcation}

The normal form of this bifurcation is known as supercritical pitchfork bifurcation. Bifurcation takes place in the point $\left(M_{x}, K_{x}, Z_{x}\right)$. The initial stable trajectory $\left(m_{0} \rightarrow M \rightarrow M_{x}\right)$ at the point $M=M_{x}$ loses its stability as the feed conversion coefficient, $Z_{x}$ grows into infinity $\left(Z_{x} \rightarrow \infty\right)$. As a result, bifurcation shows up and the following two new growth trajectories emerge (Fig.1). One emerged trajectory is $\left.\left.M_{x}\right|_{t=t_{x}} \rightarrow M_{x}\right|_{t=t_{1}}$. Second emerged trajectory is $\mathrm{M}_{\mathrm{x}} \rightarrow \mathrm{M}_{\mathrm{xx}}$, where $\mathrm{M}_{\mathrm{xx}}$ denote the species maximum weight, and the corresponding variables, $\mathrm{K}_{\mathrm{xx}}, \mathrm{t}_{\mathrm{xx}}$, and $Z_{\mathrm{xx}}$ (Stass, 2019) will be used.

In this section, we analyse bifurcation process and trajectory $\mathrm{M}_{\mathrm{x}} \rightarrow \mathrm{M}_{\mathrm{xx}}$ in detail. It should be noticed that during bifurcation, the feed conversion coefficient changes in the following mode $Z_{x} \rightarrow \infty \rightarrow Z_{x v}$, where $Z_{x v}$ denote the feed conversion coefficient on growth trajectory $\mathrm{M}_{\mathrm{x}} \rightarrow \mathrm{M}_{\mathrm{xx}}$ an instant after bifurcation. As a result, the initial point on the growth trajectory $M_{x} \rightarrow M_{x x}$ is $\left(M_{x}, K_{x}, Z_{x v}\right)$. There are two options to show this development. We start with the intuitively more obvious. Let us consider system (8).

$$
\left\{\begin{array}{l}
Z_{x x}=Z_{x} \cdot K_{x x} \\
Z_{x}=\frac{2 K_{x}\left(K_{x}-1\right)}{3} \\
K_{x}-K_{x x}=\frac{1}{K_{x}}
\end{array}\right.
$$

From the system (8), one can derive two necessary equations. The first one to consider is equation (9).

$$
\frac{\Delta K_{x x}}{\Delta K_{x}}=1-\frac{2}{3 Z_{x}}
$$

Taking limit $\left(Z_{x} \rightarrow \infty\right)$ in (9) we have 


$$
\lim _{Z_{x} \rightarrow \infty}\left(\frac{\Delta K_{x x}}{\Delta K_{x}}\right)=1
$$

We can write this result, (10) in the following form

$$
\left(Z_{x} \rightarrow \infty\right) \leftrightarrow\left(\frac{\Delta K_{x x}}{\Delta K_{x}}=1\right)
$$

Second equation to consider is (12).

$$
\frac{\Delta K_{x x}}{\Delta K_{x}}=\frac{K_{x}+1}{K_{x}}-\frac{2 K_{x}}{3 Z_{x}}
$$

Equation (12) under condition (11) takes the following form

$$
1=\frac{K_{x}+1}{K_{x}}-\frac{2 K_{x}}{3 Z_{x v}}
$$

The change from $Z_{x}$ to $Z_{x v}$ in (13) is due to the development $Z_{x} \rightarrow \infty \rightarrow Z_{x v}$. From (13) it follows

$$
Z_{x v}=\frac{2 K_{x}^{2}}{3}
$$

Numerically, $Z_{\mathrm{xv}}=69,30$ for a $600 \mathrm{~kg}$ heavy boar.

There is an obvious explanation of this result. Limit (10) has revealed the condition under which $\Delta K_{x x}=\Delta K_{x}$. This condition means that the point where trajectory $\left(\mathrm{K} \rightarrow \mathrm{K}_{\mathrm{x}}\right)$ finishes, is the same point where trajectory $\left(\mathrm{K}_{\mathrm{x}} \rightarrow\right.$ $\mathrm{K}_{\mathrm{xx}}$ ) starts. And this is the bifurcation point. In this point the development $Z_{\mathrm{x}} \rightarrow \infty \rightarrow Z_{\mathrm{xv}}$ takes place.

Second option to show the same result is the following. Let us consider equation (15).

$$
\frac{\Delta Z_{x}}{\Delta K_{x}}=\frac{2\left(K_{x}+1\right)}{3}-\frac{4 K_{x}^{2}}{9 Z_{x}}, Z_{o}=\frac{2}{3}
$$

Passage to the limit $\left(Z_{x} \rightarrow \infty\right)$ in (15) gives

$$
\lim _{Z_{x} \rightarrow \infty}\left(\frac{\Delta Z_{x}}{\Delta K_{x}}\right)=\frac{2\left(K_{x}+1\right)}{3}
$$

From (16) it follows

$$
\frac{\Delta Z_{x v}}{\Delta K_{x}}=\frac{2\left(K_{x}+1\right)}{3}, Z_{o}=\frac{2}{3}
$$

Numerically, $Z_{\mathrm{xv}}$ in (17) is 69,30 the same result as in (14).

We can summarise this section as follows.

$$
\begin{gathered}
Z_{x v}-Z_{x}=\frac{2 K_{x}}{3} \\
K_{x v}-K_{x}=0
\end{gathered}
$$

Biologically this means that the phenotypes that have passed through the bifurcation point and took on the growth trajectory $M_{x} \rightarrow M_{x x}$ start the trajectory with $Z=Z_{x v}$. The transition from $Z_{x}$ to $Z_{x v}$ occurs during bifurcation. The transition is a discrete, qualitative process that defies estimation of its time frame.

\subsection{Growth between the Bifurcation Point, and the Inflection Point}

On the growth trajectory $\mathrm{M}_{\mathrm{x}} \rightarrow \mathrm{M}_{\mathrm{xx}}$ after bifurcation, the next point to consider is an inflection point (IP). To find out the inflection point the two equations (18) and (19) have been analysed.

$$
\begin{gathered}
\mathrm{K}_{\mathrm{X}}^{2}-\mathrm{K}_{\mathrm{x}} \cdot \frac{\mathrm{M}_{\mathrm{x}}}{2 \mathrm{~m}_{\mathrm{o}}}-2=0 \\
\mathrm{Z}_{\mathrm{x}}=\frac{2 \mathrm{~K}_{\mathrm{x}}\left(\mathrm{K}_{\mathrm{x}}-1\right)}{3}
\end{gathered}
$$


There is a standard method to find out an inflection point in equation (18). Let $\mathrm{M}_{\mathrm{xxIP}}$ denote the inflection point. $M_{x x I P}=2 m_{0} K_{x}$. Numerically, $M_{x x I P}=611,769 \mathrm{~kg}$. Substituting $M_{x x I P}$ into (18) we have $K_{x x I P}$ that corresponds to $M_{x x I P .} K_{x x I P}=10,38866$. And, we can find out $Z_{x x I P}$ substituting $K_{x x I P}$ into (19). As a result, $Z_{x x I P}$ that corresponds to $\mathrm{M}_{\mathrm{xxIP}}$ numerically is $Z_{\mathrm{xxIP}}=65,02$. Let $\mathrm{t}_{\mathrm{xxIP}}$ denote the age that corresponds to $\mathrm{M}_{\mathrm{xxIP}}$. One can find it from the following condition $\left(K=K_{x x I P}\right) \wedge\left(M=M_{x x I P}\right)$. It follows $t_{x x I P}=6,652$ years. As age $t_{x}$ that corresponds to $M_{x}$ is $t_{x}=$ 6,408 years, then for $600 \mathrm{~kg}$ heavy animals, time span between the bifurcation point, and the inflection point is 89 days. In the animals, weight difference between the bifurcation point, and the inflection point is $11,769 \mathrm{~kg}$. At this IP growth rate starts to increase suggesting that between the bifurcation point, and IP growth has complicated features, since at $M_{x}$ growth has stopped. From the analyses it is reasonable to infer that during this time span changes in the growth regulation occur. Growth rate between the bifurcation point $\mathrm{M}_{\mathrm{x}}$, and IP, $\mathrm{M}_{\mathrm{xxIP}}$ is given by equation (20).

$$
\frac{1}{m_{o}} \cdot \frac{\Delta M_{x x I P \backslash x}}{\Delta t_{x x I P \backslash x}}=\frac{1}{t_{x}} \cdot \frac{2 K_{x} \cdot K_{x x I P}}{\left(K_{x}+K_{x x I P}\right)}
$$

Growth rate between IP, $\mathrm{M}_{\mathrm{xxIP}}$, and the species maximum weight, $\mathrm{M}_{\mathrm{xx}}$ is given by equation (21).

$$
\frac{1}{m_{o}} \cdot \frac{\Delta M_{x x \backslash I P}}{\Delta t_{x x \backslash I P}}=\frac{1}{t_{x x}} \cdot \frac{4 K_{x}^{2} \cdot K_{x x}}{2 K_{x} \cdot K_{x x}-K_{x x I P}^{2}}
$$

\subsection{Feed Conversion Coefficient, $Z$}

Let us consider equation (4). Equation (4) describes ontogenetic growth rate in the weight range $m_{\mathrm{o}} \leq \mathrm{M} \leq \mathrm{M}_{\mathrm{x}}$. There is no growth if

$$
\frac{\Delta M}{\Delta t}=0
$$

This is possible under condition

$$
Z(2 K+1)-2 K=0
$$

From (22) follows that $\left.Z\right|_{K=K o}=Z_{0}$. Numerically, $Z_{0}=2 / 3$. It follows that under optimal environmental and nutritional conditions animals can grow under condition that $Z_{0}>2 / 3$. This result has been used in the model.

\section{Discussion}

Our findings support the opinion that animals have systemic size regulating mechanisms, which impose a certain limit on growth, that results in a species-specific size. However, the opinion that these mechanisms are independent of growth rate and development time (Nijhout, 2015) the model does not support. Indirectly, both growth rate and development time in growth regulation are involved. This subtle, implicit involvement of the traits is due to dynamic of the feed conversion coefficient.

\subsection{Ontogenetic Growth Trajectory in Pigs}

While there has been much research on growth in pigs, bifurcation of the growth trajectory was not noticed. Bifurcation is a sudden, qualitative, discrete change in the course of the growth trajectory that causes some different trajectories to emerge. This process is inherently, in this case physiologically, conditioned. The animals that can pass through the bifurcation point take on one of some emerged growth trajectories. The ability to pass through the bifurcation point possess a limited number of animals; evidently, this quality is genetically mediated. Physiologically, this bifurcation is possible describe as the initiation of changes in the growth regulation, which concern the whole organism. In this case we can speak about the changes in which the systemic growth regulating factors are involved.

\subsection{Growth Trajectory Bifurcation}

In this section I concentrate on bifurcation details, as I believe them to be the least intuitively obvious, and for growth dynamic the most important. It should be noticed that only the animals that can attain their individual maximum weight can pass through bifurcation. It is thought that this constraint is an indication that the phenotypes are conditioned to continue to grow on the emerged trajectories. However, it is understood that the animals cannot take on one of the emerged trajectories at random; rather, this process has contingent nature. For the phenotypes, the process of taking on the right trajectory is likely genetically mediated. Bifurcation occurs when the animals attain their individual maximum weight. There is a development that causes bifurcation to show up, and that indicates that bifurcation has occurred. This development is $Z_{x} \rightarrow \infty$, during which the feed conversion coefficient 
grows into infinity. Mathematically, this is passage to the limit. An instant later, two new growth trajectories emerge.

On one trajectory $\left.\left.M_{x}\right|_{t=t_{x}} \rightarrow M_{x}\right|_{t=t_{1}}$ animals continue to live but they do not grow, so that the maximum weight remain unaffected for years (Fig.1). On this trajectory, a critical precondition of long living is the capacity to maintain individual maximum weight constant. Other relevant feature of the trajectory is that the feed conversion coefficient for all animals is equal to infinity. The two above mentioned traits, namely the capacity to maintain weight constant for years while the feed conversion coefficient is equal to infinity, are intrinsically related. This trajectory determines dynamic of growth for a set with certain phenotypes in the life stage after bifurcation. As long as the phenotypes comply with the trajectory, they can enjoy a stable and predictable life stage till the obtainable life span is attained.

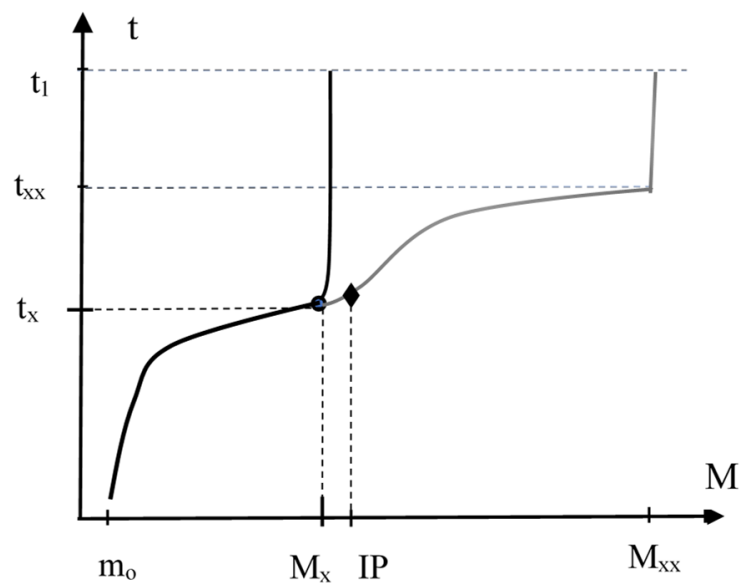

Figure 1. Pitchfork bifurcation of the growth trajectory.

- Bifurcation point $\left(\mathrm{M}_{\mathrm{x}}, \mathrm{t}_{\mathrm{x}}\right)$. Bifurcation is a sudden, qualitative, discrete change in the course of the growth trajectory that causes two different trajectories to emerge.

- Inflection point $\left(\mathrm{M}_{\mathrm{xxIP}}, \mathrm{t}_{\mathrm{xxIP}}\right)$. At this IP growth rate starts to increase.

$M_{x}$ - individual animal's maximum weight, $t_{x}$ - corresponding age. $M_{x x}$ - the species maximum weight, $t_{x x}$ corresponding age, $t_{x x}=12,69$ years. $t_{1}$ - the obtainable life span, $t_{1}=24,90$ years.

On second trajectory $\mathrm{M}_{\mathrm{x}} \rightarrow \mathrm{M}_{\mathrm{xx}}$, animals continue to grow till a species maximum weight is attained (Fig.1). This trajectory is much more complicated than the first one. On this trajectory, the growth in animals starts again and can continue till the species maximum weight is reached. The phenotypes that take on this trajectory resume the growth that has stopped at the bifurcation point. It is important to notice that the growth dynamic is completely different compared to that before bifurcation. The main point to notice is that the feed conversion coefficient, $Z_{x}$ has changed during bifurcation. Mathematically, it is one instant that separates $Z_{x}=62,51$ and $Z_{x v}=69,30$. This change in the growth rate is a shift towards a lower rate of growth at the point the growth is due to resume. It is important to notice how this change takes place. The sequence of events is the following: $\left(Z_{x} \rightarrow \infty \rightarrow Z_{x v}\right)$. In other words, the feed conversion coefficient at first grows into infinity and only then takes its initial value, $Z_{\mathrm{xv}}$ on this trajectory.

After bifurcation, the initial point on this trajectory is as follows $\left(M_{x}, K_{x}, Z_{x v}\right)$. The only visible change that has happened during bifurcation was substitution of $Z_{x}$ for $Z_{x v}$. Under the model conditions, it is enough for the trajectory to set on, and for the growth to resume. The next stage of growth is from this point $\left(M_{x}, K_{x}, Z_{x v}\right)$ up to an inflection point $\left(\mathrm{M}_{\mathrm{xxIP}}\right)$. The inflection point on the growth trajectory is the point at which the trajectory's dynamic changes. At this inflection point, growth rate starts to increase (Fig.1). Before the inflection point, it is from an instant after bifurcation up to the inflection point the growth rate seems does not increase. This period of growth for $600 \mathrm{~kg}$ heavy boar takes 89 days. The growth during this time span has an unusual feature. In this growth stage, the rate of growth seems has tendency to decrease. This growth dynamic looks like an impetus, which was received at the bifurcation point and that gradually dissipates towards the inflection point. It is feasible that during this time span the physiological changes that concern growth regulation to comply with the trajectory take place. At the inflexion point, the growth rate starts to increase, and the animals continue to grow up to the species maximum weight, $\mathrm{M}_{\mathrm{xx}}$ is attained. 
When the species maximum weight, $\mathrm{M}_{\mathrm{xx}}$ has been attained, animals' ontogenetic growth stops. As a result, the feed conversion coefficient, $Z_{x x}$ grows into infinity $\left(Z_{x x} \rightarrow \infty\right)$. This development takes place at $M=M_{x x}, K=K_{x x}$, and age $t_{x x}=12,69$ years (Stass, 2017). These phenotypes can continue to live till obtainable life span is attained, provided their individual maximum weight remain constant. In this relevant aspect, the two trajectories $(M \rightarrow$ $\left.\left.\left.M_{x}\right|_{t=t_{x}} \rightarrow M_{x}\right|_{t=t_{1}}\right)$ and $\left(\left.\left.M \rightarrow M_{x x}\right|_{t=t_{x x}} \rightarrow M_{x x}\right|_{t=t_{1}}\right)$ have nontrivial similarities (Fig.1). It follows that the option to attain the obtainable life span is to maintain the individual maximum weight or the species maximum weight constant for years. Under the model conditions, stability of the weight is an essential trait related to longevity. It is reasonable to infer that in the animals, there is a trade-off between live weight, life span, and feed conversion.

In general terms, this bifurcation is possible describe as follows. Before bifurcation, animals grow on a continuous, phenotype-dependent trajectory. The phenotypes that cannot reach their maximum weight (Stass, 2019) cannot enter this bifurcation. At the point the animals attain their individual maximum weight, initial trajectory loses its stability as $Z_{x}$ grows into infinity $\left(Z_{x} \rightarrow \infty\right)$. As a result, bifurcation shows up and two new growth trajectories emerge. In terms of systems theory, this is supercritical pitchfork bifurcation. The emerged trajectories are asymptotically, but not globally, stable. This means that as long as phenotypes can comply with the trajectory, they can enjoy a predictable life stage. In other words, the emerged trajectories are genetic channels that open the way to grow for the phenotypes that can comply with the conditions. These channels lead to the obtainable life span. Under the model conditions this is the advance that enables the phenotypes to attain the species obtainable life span. In this study, we talk about animals and it is feasible that the animals take on one of the emerged trajectories contingent on phenotype, and not at random. Similar analyses of growth in humans would be of big advantage for research on senescence and life span.

\subsection{Feed Conversion Coefficient, $Z$}

The feed conversion coefficient is an intricate trait. It was not accounted for in many studies. The trait is also highly complex in nature, because it is affected by much more than diet composition (Patience et al. 2015). While there has been extensive research on the trait, the trait's dynamic in animals' ontogeny is poorly understood. An important aspect of feed conversion coefficient is that this trait is not functional throughout all life span in animals. Many animals, after attainment of maximum individual weight can live for years with the feed conversion coefficient equal to infinity. Besides, in systems dynamic terms, the feed conversion coefficient $Z$, is the order parameter; it causes the growth trajectory to bifurcate at $Z=Z_{x}$, and $Z=Z_{x x} . Z$ is always positive, and may be less than or greater than 1 contingent on growth phenotype and growth conditions. Animals can grow if $Z>2 / 3$ and $Z$ $\neq \infty$.

This study offers a reading of the dynamic of the feed conversion coefficient in pigs' ontogeny. In animals, live weight increase $\left(m_{0} \rightarrow M \rightarrow M_{x}\right)$ corresponds to $\left(Z_{0} \rightarrow Z \rightarrow Z_{x} \rightarrow \infty\right)$, and $\left(m_{0} \rightarrow M \rightarrow M_{x} \rightarrow M_{x x}\right)$ corresponds to $\left(Z_{0} \rightarrow Z \rightarrow Z_{x} \rightarrow \infty \rightarrow Z_{x v} \rightarrow Z_{x x I P} \rightarrow Z_{x x} \rightarrow \infty\right)$. This dynamic is nonlinear and as yet poorly understood.

It should be noticed that $Z_{x}$ is unstable and exists only one instant, the bifurcation instant. This means that $Z_{x}$ is not observable and can only be calculated. The relation between $Z_{x}$ and $Z_{x v}$ is the following

$$
Z_{x v}-Z_{x}=\frac{2 K_{x}}{3}
$$

\subsection{Invariant of Growth, Parameter K}

Growth modelling depends critically on the variables that have been applied for the task to complete. In this respect, parameter $\mathrm{K}$ is a focal variable introduced to model growth in animals. Parameter $\mathrm{K}$ is the growth invariant, it is the same for the same weight animals. Parameter $\mathrm{K}$ is a function of the current weight, $\mathrm{M}$ that is a continuous variable, and current time, $\mathrm{t}$ which is a discrete variable. Both hybrid quality of the parameter $\mathrm{K}$ and hybrid formulations of the weight dynamic make the model an appropriate tool for studying growth in animals. As a result, it imposes constraints on functional relations between the variables that are used in the model. Parameter $\mathrm{K}$ is species-specific; it serves to model ontogenetic growth in domestic pigs. The fact that parameter $\mathrm{K}$ is species-specific, makes the whole model species-specific. This results from the constraints that parameter $\mathrm{K}$ imposes on other variables. It has the consequence that parameter $\mathrm{K}$ is a relevant variable in the model. In the animals' ontogeny, the dynamic of parameter $\mathrm{K}$ is as follows. On one trajectory $\left(\mathrm{K}_{0} \rightarrow \mathrm{K} \rightarrow \mathrm{K}_{\mathrm{x}} \rightarrow \mathrm{K}_{1}\right)$, on other trajectory $\left(\mathrm{K}_{\mathrm{o}} \rightarrow \mathrm{K} \rightarrow \mathrm{K}_{\mathrm{x}} \rightarrow \mathrm{K}_{\mathrm{xxIP}} \rightarrow \mathrm{K}_{\mathrm{xx}} \rightarrow \mathrm{K}_{1}\right)$.

\section{Conclusions}


$\circ$ In the course of the ontogenetic growth, bifurcation of the growth trajectory is a regular process. As a result, two new growth trajectories emerge. Bifurcation initiates changes in the growth regulation that concern the whole organism. The changes in the growth regulation occur in 89 days while animals grow between the bifurcation point, and the inflection point.

$\circ$ During bifurcation, a change from $Z_{x}$ to $Z_{x v}$ occurs in the following mode $Z_{x} \rightarrow \infty \rightarrow Z_{x v}$. This change in the feed conversion coefficient is a discrete, intrinsically determined switch in the course of the growth trajectory.

$\circ Z_{x}$ is unstable and exists only one instant, the bifurcation instant. This means that $Z_{x}$ is not observable in experiments; it can only be calculated, and analysed in mathematical models.

$\circ$ In animals' ontogeny, the feed conversion coefficient, $Z$ is the order parameter; it causes the growth trajectory to bifurcate, and it modulates growth rate and the rate of ontogeny. Animals can grow if $Z>2 / 3$ and $Z \neq \infty$. Ontogenetic growth stops when the feed conversion coefficient grows into infinity.

○ Through the bifurcation point can pass only some phenotypes. These phenotypes are conditioned to take on one of the emerged trajectories. The phenotypes are thought take on the trajectories in close accordance with their genetic determinants.

○ After bifurcation, as long as the phenotypes can comply with their trajectory, they can enjoy a stable and predictable life stage.

- The emerged trajectories are genetic channels that open the way to grow for the certain phenotypes. Under the model conditions, these trajectories are the channels that enable the phenotypes to attain the species life span.

○ In animals' ontogeny, three sets with growth phenotypes are observed. One set with phenotypes that cannot reach their individual maximum weight. One set with phenotypes that grow on trajectory $\left.M \rightarrow M_{x}\right|_{k=k_{x}} \rightarrow$ $\left.M_{x}\right|_{k=k_{1}}$. And one set with phenotypes that grow on trajectory $\mathrm{M} \rightarrow \mathrm{M}_{\mathrm{x}} \rightarrow \mathrm{M}_{\mathrm{xx}}$.

○ In ontogeny, the stability of weight is an essential trait related to longevity. The condition to attain obtainable life span is to maintain maximum weight constant for years. Research suggests that in pigs there is a trade-off between live weight, life span, and feed conversion.

\section{Conflict of interests}

The authors declare that there is no conflict of interests regarding the publication of this paper.

\section{References}

Aihara, K., \& Suzuki, H. (2010). Theory of hybrid dynamical systems and its applications to biological and medical systems. Phil. Trans. R. Soc. A, 368, 4893-4914.

Aumann, C. A. (2006). A methodology for developing simulation models of complex systems. Ecological Modelling, 202, 385-396.

Brenner, S. (2010). Sequences and consequences. Phil. Trans. R. Soc. B, 365, 207-212.

Coveney, P. V., \& Fowler, P. W. (2005). Modelling biological complexity: A physical scientist's perspective. J. R. Soc. Interface, 2, 267-280.

Coveney, P. V., Dougherty, E. R., \& Highfield, R. R. (2016). Big data need big theory too. Phil. Trans. R. Soc. A, 374, 20160153.

Di Bernardo, M., \& Hogan, S. J. (2010). Discontinuity-induced bifurcations of piecewise smooth dynamical systems. Phil. Trans. R. Soc. A, 368, 4915-4935.

Louzoun, Y., Solomon, S., Atlan, H., \& Cohen, I. R. (2001). Modeling complexity in biology. Physica A, 297, 242-252.

Lui, J. C., \& Baron, J. (2011). Mechanisms Limiting Body Growth in Mammals. Endocrine Reviews, 32, 422-440.

Lunney, J. K. (2007). Advances in Swine Biomedical Model Genomics. International Journal of Biological Sciences, 3(3),179-184.

Maini, P. K., Wooley, T. E., Baker, R. E., Gaffney, E. A., \& Lee, S. (2012). Turing's model for biological pattern formation and the robustness problem. Interface Focus, 2, 487-496.

Nijhout, H. F. (2015). Big or fast: Two strategies in the developmental control of body size. BMC Biology, 13, 57.

Noble, D. (2002). Modeling the Heart - from Genes to Cells to the Whole Organ. SCIENCE, 295, 1678-1682. 
Osborne, J. M., Walter, A., Kershaw, S. K., Mirams, G. R., Fletcher, A. G., Pathmanathan, P., Gavaghan, D., Jensen, O. E., Maini, P. K., \& Byrne, H. M. (2010). A hybrid approach to multi-scale modelling of cancer. Phil. Trans. R. Soc. A, 368, 5013-5028.

Patience, J. F., Rossoni-Serão, M. C., \& Gutiérrez, N. A. (2015). A review of feed efficiency in swine: Biology and application. Journal of Animal Science and Biotechnology, 6, 33.

Schumacher, L. J., Kulesa, P. M., McLennan, R., Baker, R. E., \& Maini, P. K. (2016). Multidisciplinary approaches to understanding collective cell migration in developmental biology. Open Biol., 6, 160056.

Stass, V. L. (2017). An analytical model of animal growth. European Scientific Journal, 13(27). 1-17.

Stass, V. L. (2019). Genetic potential for growth, feed conversion and longevity. International Journal of Biology, $11(1) .66-75$.

Stewart, I. (2011). Sources of uncertainty in deterministic dynamics: an informal overview. Phil. Trans. R. Soc. A., $369,4705-4729$.

Weinkove, D., \& Leevers, S. J. (2000). The genetic control of organ growth: Insights from Drosophila. Current Opinion in Genetics \& Development, 10, 75-80.

\section{Copyrights}

Copyright for this article is retained by the author(s), with first publication rights granted to the journal.

This is an open-access article distributed under the terms and conditions of the Creative Commons Attribution license (http://creativecommons.org/licenses/by/4.0/). 